\title{
MCMC-Based Fatigue Crack Growth Prediction on 2024-T6 Aluminum Alloy
}

\author{
Xu Du, ${ }^{1}$ Yu-ting He, ${ }^{1}$ Chao Gao, ${ }^{1,2}$ Kai Liu, ${ }^{1}$ Teng Zhang, ${ }^{1}$ and Sheng Zhang ${ }^{1}$ \\ ${ }^{1}$ Aeronautics and Astronautics Engineering College, Air Force Engineering University, Xi'an 710038, China \\ ${ }^{2}$ Beijing Aeronautical Technology Research Center, Beijing 100076, China \\ Correspondence should be addressed to Yu-ting He; heyut666@126.com
}

Received 31 May 2017; Accepted 22 August 2017; Published 3 October 2017

Academic Editor: Yuqiang Wu

Copyright (c) $2017 \mathrm{Xu}$ Du et al. This is an open access article distributed under the Creative Commons Attribution License, which permits unrestricted use, distribution, and reproduction in any medium, provided the original work is properly cited.

\begin{abstract}
This work aims to make the crack growth prediction on 2024-T6 aluminum alloy by using Markov chain Monte Carlo (MCMC). The fatigue crack growth test is conducted on the 2024-T62 aluminum alloy standard specimens, and the scatter of fatigue crack growth behavior was analyzed by using experimental data based on mathematical statistics. An empirical analytical solution of Paris' crack growth model was introduced to describe the crack growth behavior of 2024-T62 aluminum alloy. The crack growth test results were set as prior information, and prior distributions of model parameters were obtained by MCMC using OpenBUGS package. In the additional crack growth test, the first test point data was regarded as experimental data and the posterior distribution of model parameters was obtained based on prior distributions combined with experimental data by using the Bayesian updating. At last, the veracity and superiority of the proposed method were verified by additional crack growth test.
\end{abstract}

\section{Introduction}

In aircraft structural engineering domain, the concept of damage tolerance was introduced to design key components. The prediction of fatigue crack propagation is a main work for aircraft structure service/use life management.

Accurate prediction of fatigue crack growth determined the safety of service and usage. Due to the scatter of material, the fatigue crack growth behavior of some components still creates scatter, though the components made by the same material and served under the same loading and environmental conditions.

The residual crack propagation life of the aircraft components is assessed based on the in-service observation and the mathematical model which describes the crack growth behavior of these components. The input data provided to these mathematical models are generally uncertain. There are uncertainties in the material performance, components dimensions, the measurements, and the degradation model [1]. Probability methodology is widely used to make decision by modelling these uncertainties with suitable probability distribution function. Scholars around the world have done a lot of work on the scatter of fatigue crack growth [2-4]. In addition, some stochastic fatigue crack growth models have been proposed $[5,6]$.

The experimental results used to characterize the model parameters can be regarded as the prior information, and it has an associated prior probability distribution function. The in-service inspection data can be used as experimental observation, which conjuncts the prior distribution of the model parameters to reduce the uncertainty in the prediction of fatigue crack growth curve for one component [7]. The posterior distribution can be used to make updated estimates of model parameter for the crack growth behavior.

The combination of prior distribution of fatigue crack growth model parameters with experimental observations can be carried out by applying the concepts of Bayesian updating. The results from updated distribution function are also termed as posterior distribution. The Markov chain Monte Carlo (MCMC) is one of the common probability simulation methods through the Bayesian updating [8]. And the Bayesian updating can be accomplished by OpenBUGS package. 
TABLE 1: Chemical compositions of 2024-T62 (\%).

\begin{tabular}{cccccccccc}
\hline $\mathrm{Cu}$ & $\mathrm{Mg}$ & $\mathrm{Mn}$ & $\mathrm{Si}$ & $\mathrm{Fe}$ & $\mathrm{Zn}$ & $\mathrm{Ti}$ & $\mathrm{Cr}$ & Other & $\mathrm{Al}$ \\
\hline 4.64 & 1.49 & 0.68 & $<0.5$ & $<0.5$ & $<0.25$ & $<0.15$ & $<0.1$ & $<0.15$ & Balance \\
\hline
\end{tabular}

TABLE 2: Mechanical performance of 2024-T62 (\%).

\begin{tabular}{lccc}
\hline$E / \mathrm{GPa}$ & $\sigma_{b} / \mathrm{MPa}$ & $\sigma_{0.2} / \mathrm{MPa}$ & $\zeta_{5} / \%$ \\
\hline 71 & 451 & 400 & 7.2 \\
\hline
\end{tabular}

In this study, fatigue crack growth tests of 2024-T62 aluminum alloy were conducted under the constant amplitude loading. The prior distribution of model parameters was obtained based on experimental $a-N$ data. Afterwards, a new fatigue crack growth prediction approach was proposed based on the prior distribution, in-service observation, and Bayesian updating method. Finally, the proposed approach is validated by the additional fatigue crack growth under the same test conditions.

\section{Fatigue Crack Propagation Test}

The 2024-T62 aluminum alloy is widely used as the main load structure in Chinese aviation industry.

2.1. Material and Specimen. Chemical compositions and mechanical performance of 2024-T62 aluminum alloy are shown in Tables 1 and 2, respectively.

With a hole in the center throughout whole specimen, it has been machined from the 2024-T62 aluminum alloy pipe material along the $L-T$ direction. The dimensional length of specimen is shown in Figure 1, the thickness is $2.5 \mathrm{~mm}$, and the diameter of center hole is $4 \mathrm{~mm}$. The specimen was designed and conducted according to the ASTM standard E647 requirements [9].

2.2. Experimental Procedure. All the tests were carried out with the loading frequency of $20 \mathrm{~Hz}$ using a servo hydraulic universal dynamical test machine (MTS-810-500KN). All the tests were conducted under the room temperature $\left(20^{\circ} \mathrm{C}\right)$. In the test, the measurements of the crack length use the Questar QM1 long working distance microscope (limit of resolution is $2.7 \mu \mathrm{m})$.

When the crack approximately propagates to $0.5 \mathrm{~mm}$, start to count the number of loading cycle. At the same time, the crack length was measured by per 9000 loading cycles. It is worth noting that $0.5 \mathrm{~mm}$ is called initial crack length.

2.3. Experimental Results. The experimental fatigue crack propagation length from number 1 to number 4 is listed in Table 3. It is well known that the crack growth propagation region can be divided to three regions: near-threshold $\left(\Delta K_{\mathrm{th}}\right)$ region, stable crack propagation region, and unstable (fast) crack propagation region [10]. In order to obtain more stable data, the stable crack propagation region was chosen (crack length: $0.5 \mathrm{~mm} \sim 6 \mathrm{~mm}$ ).

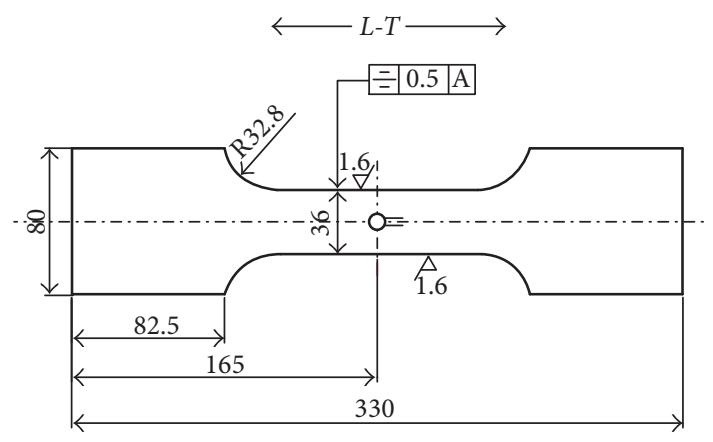

FIGURE 1: Geometry length of specimen.

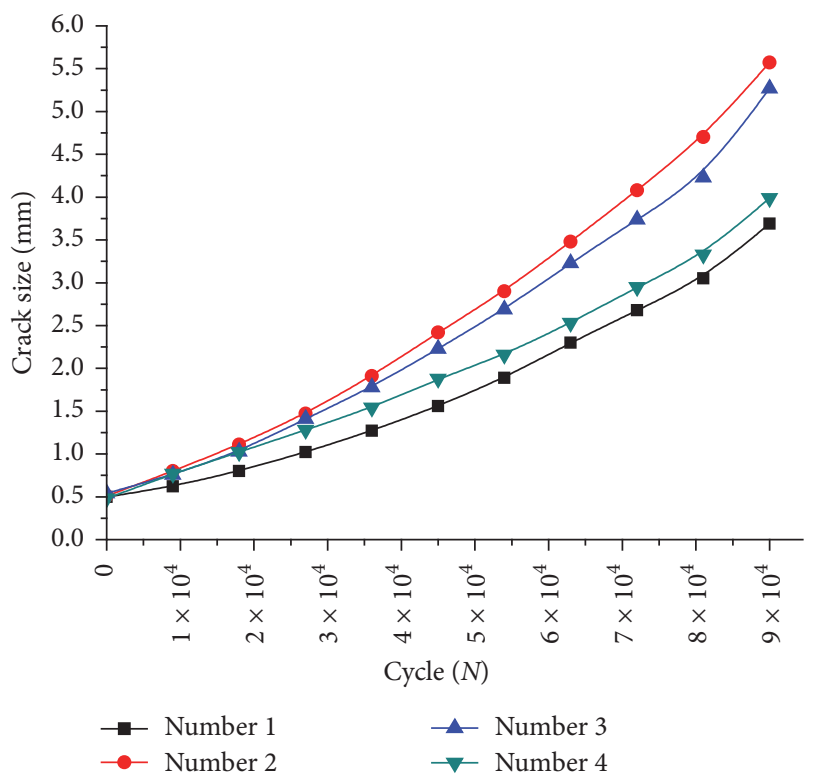

FIGURE 2: Experimental fatigue crack growth $a-N$ curve.

The experimental fatigue crack propagation $a-N$ curves for four specimens are shown in Figure 2.

As shown in Figure 2, at a given cycles, crack length has a wide range of scatter even under the same loading condition and test condition. The scatter of crack growth may be mainly attributed to the inhomogeneous material properties. Therefore, it is necessary to investigate the scatter of fatigue crack growth behavior.

\section{Statistical Analyses of Crack Growth Data}

The scatter of 2024-T62 aluminum alloy is analyzed by statistical analysis method. And crack growth behavior of 2024T62 aluminum alloy is described by mathematical model.

3.1. Scatter Analysis of Crack Growth Behavior. The mean value $\bar{x}$, standard deviation $\sigma$, and coefficients of variation (COV) of specimens sample are common statistical parameters to describe the variation of statistics. Mean value is used to describe the centralized location of sample data, but $\sigma$ and $\mathrm{COV}$ are used to describe the scatter. 
TABLE 3: Crack lengths for different cycles (mm).

\begin{tabular}{|c|c|c|c|c|c|c|c|c|c|c|c|}
\hline \multirow{2}{*}{ Specimen } & \multicolumn{11}{|c|}{ Crack length } \\
\hline & 0 & 9000 & 18000 & 27000 & 36000 & 45000 & 54000 & 63000 & 72000 & 81000 & 90000 \\
\hline No. 1 & 0.5 & 0.62 & 0.8 & 1.02 & 1.27 & 1.56 & 1.89 & 2.3 & 2.68 & 3.05 & 3.69 \\
\hline No. 2 & 0.51 & 0.8 & 1.11 & 1.47 & 1.91 & 2.42 & 2.9 & 3.48 & 4.08 & 4.7 & 5.57 \\
\hline No. 3 & 0.54 & 0.76 & 1.03 & 1.41 & 1.78 & 2.23 & 2.69 & 3.23 & 3.74 & 4.23 & 5.27 \\
\hline No. 4 & 0.48 & 0.77 & 1.02 & 1.28 & 1.54 & 1.88 & 2.16 & 2.53 & 2.95 & 3.33 & 3.99 \\
\hline
\end{tabular}

TABLE 4: Statistics variables of crack lengths for different cycles.

\begin{tabular}{lcccccccccc}
\hline \multirow{2}{*}{ Statistics variables } & \multicolumn{10}{c}{ Cycles } \\
& 9000 & 18000 & 27000 & 36000 & 45000 & 54000 & 63000 & 72000 & 81000 & 90000 \\
\hline$\mu$ & 0.7375 & 0.99 & 1.295 & 1.625 & 2.0225 & 2.41 & 2.885 & 3.3625 & 3.8275 & 4.63 \\
$\sigma$ & 0.0870 & 0.1443 & 0.2168 & 0.3060 & 0.4134 & 0.5058 & 0.6080 & 0.7126 & 0.8350 & 1.0078 \\
COV & 0.1087 & 0.1343 & 0.1542 & 0.1735 & 0.1883 & 0.1934 & 0.1942 & 0.1953 & 0.2010 & 0.2005 \\
\hline
\end{tabular}

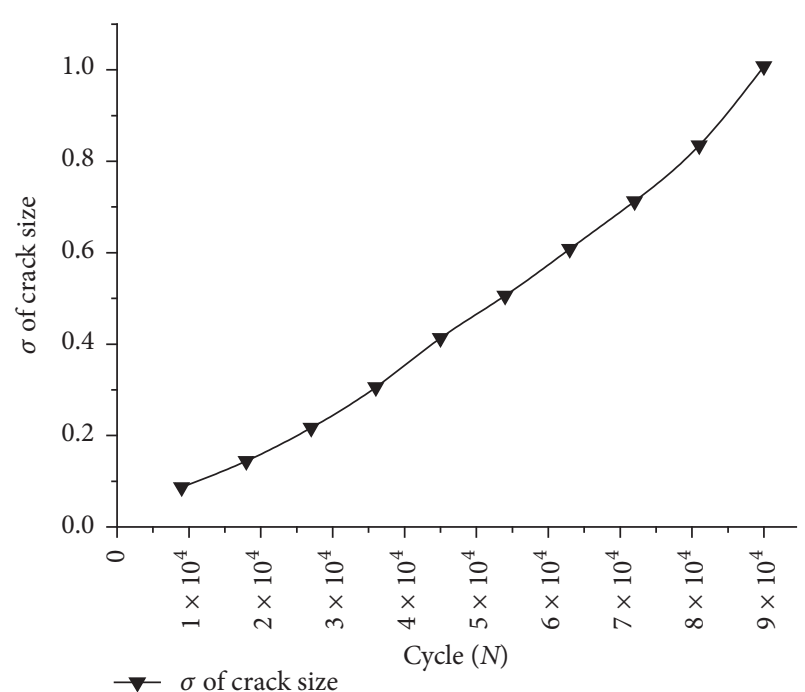

(a)

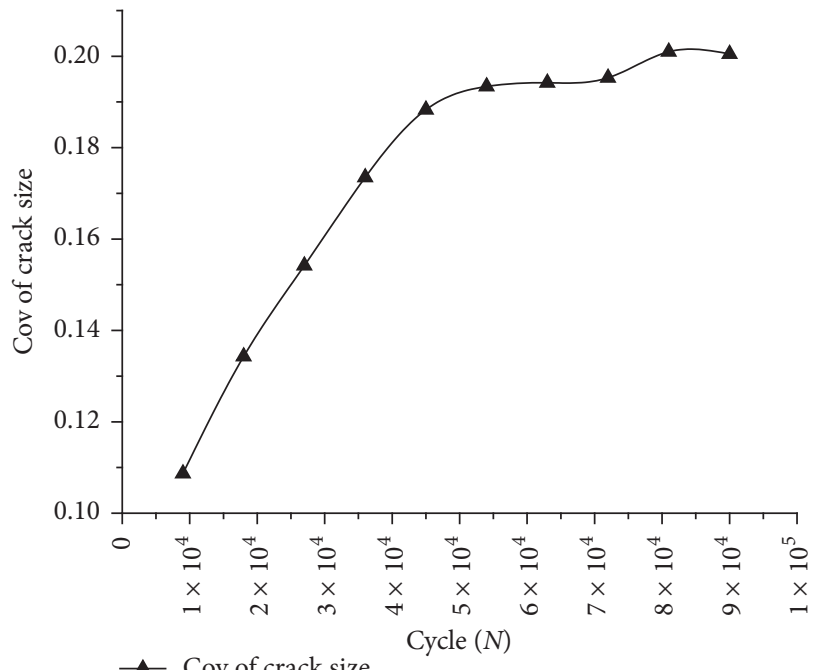

(b)

FIGURE 3: Change trend of statistical variables: (a) standard deviation $\sigma$ and (b) coefficients of variation COV.

Mean value and standard deviation of observation $x_{1}, x_{2}, \ldots, x_{n}$ can be expressed as

$$
\begin{aligned}
& \bar{x}=\frac{1}{n}\left(x_{1}+x_{2}+\cdots+x_{n}\right) \\
& \sigma=\sqrt{\frac{\sum_{i=1}^{n} x_{i}^{2}-n \bar{x}^{2}}{n-1}} .
\end{aligned}
$$

The coefficients of variation COV (simply as $C$ ) are shown as

$$
C=\frac{s}{\bar{x}} \text {. }
$$

The statistics $\mu, \sigma$, and COV were calculated based on experimental results (in Table 4). The statistical calculating formulas ((1) and (2)) are listed in Table 4.

Figure 3 gives the trend of standard deviation and COV of crack length (under same loading cycles).
As listed in Table 4 and depicted in Figure 3, standard deviation $\sigma$ and coefficients of variation COV increase gradually with loading cycles increase. In a word, the scatter of crack growth process increases gradually.

3.2. Statistics of Fatigue Crack Growth Behavior. The fatigue crack growth (FCG) is modelled using Paris' law [11]. Paris' law gives the crack growth rate per stress cycle as a function of the range of stress intensity factor and material constant.

$$
\frac{d a}{d N}=C(\Delta K)^{m},
$$

where $a$ is the crack length (mm), $N$ is number of loading cycles, $d a / d N$ is the crack growth rate per loading cycle due to fatigue, $\Delta K$ is the range of stress intensity factor (in Mpa $\sqrt{\mathrm{m}}$ ), and $C$ and $m$ are constant material properties obtained by testing standard specimens made of this material. 
TABLE 5: Estimation value of model parameters.

\begin{tabular}{lcc}
\hline \multirow{2}{*}{ Specimen } & \multicolumn{2}{c}{ Parameter estimate value } \\
& $\theta_{1}$ & $\theta_{2}$ \\
\hline Number 1 & 2.4312 & -0.2598 \\
Number 2 & 3.7303 & -0.5435 \\
Number 3 & 3.2953 & -0.4592 \\
Number 4 & 3.0991 & -0.7331 \\
\hline
\end{tabular}

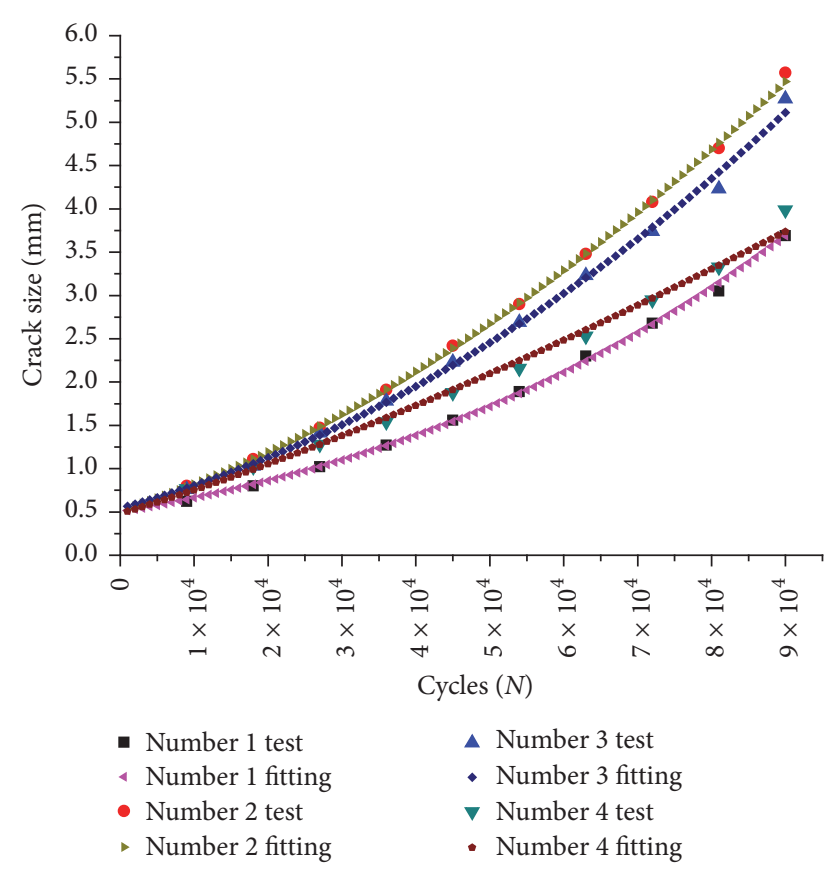

FIGURE 4: Fitting curves versus experimental results.

The crack length after $N$ number of loading cycles is $a(N)$. The crack growth rate $d a / d N$ determines the true crack length $a(N)$ according to Paris' law (3), which has the form of $\theta_{1} k(a)^{\theta_{2}+1}$, for some function $k(a)$. When $k(a)=a$, the solution for $a(N)$ can be expressed by [12]

$$
a(N)=\frac{a_{0}}{\left(1-a_{0} \theta_{2} \theta_{1} \theta_{2} N\right)^{1 / \theta_{2}}},
$$

where $a_{0}$ is the initial crack length (in mm). $\theta_{1}$ and $\theta_{2}$ are the parameters of crack growth model for 2024-T62 aluminum alloy under constant amplitude loading. So (4) is an empirical analytical solution form for Paris' crack growth law.

Firstly, it is necessary to validate the feasibility of using (4), which can describe the crack growth behavior of 2024T62 aluminum alloy. The estimation value of $\theta_{1}$ and $\theta_{2}$ can be obtained based on crack growth experimental $a-N$ data by maximum likelihood method (MLE). The values of model parameters $\theta_{1}$ and $\theta_{2}$ for four specimens are listed in Table 5 .

The fatigue crack growth curve can be obtained by substituting the estimation value of model parameters $\theta_{1}$ and $\theta_{2}$ to (4). And the obtained curve is the fitting curve of crack growth, which is shown in Figure 4.

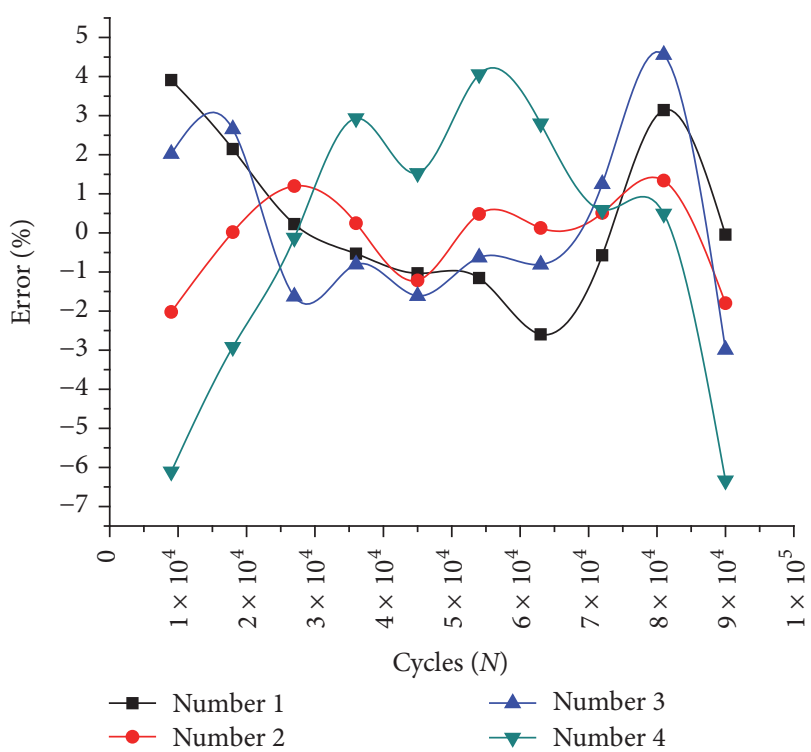

FIGURE 5: Error between experimental data and fitting curves.

It can be seen from Figure 4 that the fitting curves (based on (4)) are in good agreement with the experimental results, which can validate the feasibility of (4).

Relative error (crack growth fitting curves to experimental data) of four specimens is listed in Figure 5 and Table 6.

As listed in Table 6 and shown in Figure 5, the relative errors between the fitting curves and the experimental results were less than $7 \%$. It means that the approach (include (4) and parameter estimating method) could be satisfactorily used in engineering. As a result, (4) is a feasible and accurate model to describe the crack growth behavior of 2024-T62 aluminum alloy under constant amplitude loads.

\section{Prediction Method of Fatigue Crack Growth}

In aircraft structural engineering, the common use of the media crack growth $a-N$ experimental results represents the fatigue crack propagation behavior, which cannot consider the scatter and the distinguish of different specimens.

4.1. Prior Distribution. As we all know, crack length $a(N)$ is a nonlinear function of loading cycles numbers $N$. By taking natural logarithms $e$ in (4), we can obtain the transformed real crack length $\ln \left[a(N) / a_{0}\right]$ as

$$
\ln \left[\frac{a(N)}{a_{0}}\right]=-\frac{1}{\theta_{2}} \ln \left(1-a_{0}{ }^{\theta_{2}} \theta_{1} \theta_{2} N\right) .
$$

For the experimental observed transformed crack length $Y$, it includes the measure error $\varepsilon$. And every $Y_{i j}$ can be expressed as

$$
Y_{i j}=-\frac{1}{\theta_{2 i}} \ln \left(1-a_{0 i}{ }^{\theta_{2 i}} \theta_{1 i} \theta_{2 i} N_{i j}\right)+\varepsilon_{i j},
$$

where subscripts $i$ and $j$ represent sequence number of specimen and sequence number of experimental observations point, respectively. So $Y_{i j}$ is the observed transformed 
TABLE 6: Parameter Estimate of fatigue crack propagation curve.

\begin{tabular}{lcccccccccc}
\hline \multirow{2}{*}{ Specimen } & \multicolumn{10}{c}{ Error (\%) } \\
& 9000 & 18000 & 27000 & 36000 & 45000 & 54000 & 63000 & 72000 & 81000 & 90000 \\
\hline Number 1 & $3.91 \%$ & $2.15 \%$ & $0.22 \%$ & $-0.53 \%$ & $-1.04 \%$ & $-1.16 \%$ & $-2.60 \%$ & $-0.57 \%$ & $3.14 \%$ & $-0.04 \%$ \\
Number 2 & $-3.95 \%$ & $-1.94 \%$ & $-0.79 \%$ & $-1.72 \%$ & $-3.16 \%$ & $-1.49 \%$ & $-1.84 \%$ & $-1.46 \%$ & $-0.65 \%$ & $-3.72 \%$ \\
Number 3 & $-5.53 \%$ & $-4.95 \%$ & $-8.91 \%$ & $-8.15 \%$ & $-8.90 \%$ & $-7.99 \%$ & $-8.16 \%$ & $-6.25 \%$ & $-3.19 \%$ & $-10.18 \%$ \\
Number 4 & $-2.20 \%$ & $1.13 \%$ & $4.04 \%$ & $7.22 \%$ & $5.76 \%$ & $8.39 \%$ & $7.08 \%$ & $4.78 \%$ & $4.68 \%$ & $-2.43 \%$ \\
\hline
\end{tabular}

crack length for $j$ th observation point of $i$ th specimen. And $a_{0 i}$ is the initial crack length of $i$ th specimen. $\varepsilon_{i j}$ is the observation error for $j$ th observation point of $i$ th specimen, which is subjected to normal distribution $\left(N\left(0, \sigma^{2}\right)\right)$. And error $\varepsilon_{i j}$ for different specimens and different observations is independent identically distribution (i.i.d.).

The scatter of fatigue crack growth behavior in the model parameter is modelled by using a prior probability density function (PDF). And the assumption model parameters $\vec{\theta}_{i}=\left(\theta_{1 i}, \theta_{2 i}\right)$ subjected to multinormal distribution can be expressed as

$$
\vec{\theta}_{i} \sim \operatorname{multi-Normal}(\vec{\mu}, \vec{\Sigma})
$$

Likelihood of experimental transformed crack length includes the PDF of $y_{i j}$ and the PDF of model matrix parameter $\vec{\theta}_{i}=\left(\theta_{1 i}, \theta_{2 i}\right)$.

$$
y_{i j} \sim \operatorname{Normal}\left[-\left(\frac{1}{\theta_{2 i}}\right) \ln \left(1-a_{0 i}{ }^{\theta_{2 i}} \theta_{1 i} \theta_{2 i} N_{i j}\right), \sigma^{2}\right] \text {. }
$$

In case of completely lack prior information, the diffuse distribution can be used to describe the prior information that can also be called noninformative prior distribution [13]. Assumption matrix parameter $\vec{\mu}$ subjected to multinormal distribution can be expressed as

$$
\begin{aligned}
\vec{\mu} \sim \operatorname{multi-Noraml}( & \left.\vec{\mu}, \vec{\Sigma}_{\mu 0}\right), \\
& \vec{\mu}_{0}=\left(\begin{array}{l}
0 \\
0
\end{array}\right) \vec{\Sigma}_{\mu 0}=\left(\begin{array}{cc}
1000 & 0 \\
0 & 1000
\end{array}\right) .
\end{aligned}
$$

Assumption parameter $\vec{\Sigma}$ subjected to inverse Wishart distribution can be expressed as

$$
\vec{\Sigma} \sim \text { InverseWishart }\left(\vec{\Sigma}_{0}, 2\right), \quad \vec{\Sigma}_{0}=\left(\begin{array}{cc}
10 & 0 \\
0 & 10
\end{array}\right) .
$$

And assumption parameter $\sigma^{2}$ subjected to inverse Gamma distribution is expressed as

$$
\sigma^{2} \sim \text { InverseGamma }(3,0.001) .
$$

$\mu_{1}, \mu_{2}, \Sigma_{11}, \Sigma_{12}, \Sigma_{21}, \Sigma_{22}$, and $\sigma$ are the parameters of fatigue crack growth model. The posterior PDF of the model parameter is obtained using Bayesian updating, implemented using the MCMC algorithms. MCMC algorithms are a general class of computational methods used to produce samples from posterior samples. They are often easy to implement and, at least in principle, can be used to simulate from very high-dimensional posterior distributions [12]. And they have been successfully applied to literally thousands of applications. Metropolis-Hastings algorithms and Gibbs samplers are two general categories of MCMC simulation.

Briefly, OpenBUGS is a software package for Bayesian analysis of complex statistical models with the Gibbs sampler, which is a based implementation of BUGS (Bayesian Inference Using Gibbs Sample). The package contains flexible software for analyzing complex statistical models using MCMC methods. For more information about background, application, and introduction to OpenBUGS refer to Amiri and Modarres [14] and Spiegelhalter et al. [15] and Cowles. The model parameters statistics estimated from tested data (in Table 3) and above method are listed in Table 7.

One way to assess the accuracy of the posterior estimation is by calculating the Monte Carlo error (MC-error) for each parameter. This is an estimate for the difference between the mean of the sampled values (which we are using as our estimate of the posterior mean for each parameter) and the true posterior mean.

As a rule of thumb, the simulation should be run until the Monte Carlo error for each parameter of interest is less than about $5 \%$ of the sample standard deviation. As listed in Table 7, the MC-error for each parameter is all less than $5 \%$. Therefore, we can know that the posterior distribution of model parameter is accurate.

4.2. Convergence Diagnose. Convergence diagnosis is an important work in the using of MCMC algorithm. If the convergence feature of chains is poor, the posterior distributions from chains based on MCMC are not accurate. Figure diagnosis method, checking throughout mean method, and contrasting deviation method are common methods to diagnose the convergence. And contrasting deviation method has a wide use in the convergence diagnosis analysis.

Based on the contrasting deviation method proposed by Gelman and Rubin [16], a more brief and convenient method was established by Brooks and Gelman [17]. The basic idea is to generate multiple chains starting at overdispersed initial values and assess convergence by comparing within-chain and between-chain variability over the second half of those chains. We denote the number of chains generated by $L$ and the length of each chain by $2 T$. We take as a measure of posterior variability the width of the $100(1-\alpha) \%$ credible 
TABLE 7: Parameter Estimate of fatigue crack propagation curve.

\begin{tabular}{lcccccccc}
\hline \multirow{2}{*}{ Parameter } & \multirow{2}{*}{ Mean } & \multirow{2}{*}{ Standard deviation } & \multirow{2}{*}{ MC-error (\%) } & \multicolumn{3}{c}{ Different percentile } \\
& & & & 0.025 & 0.05 & 0.5 & 0.95 & 0.975 \\
\hline$\mu_{1}$ & 3.1390 & 0.3344 & 0.09 & 2.4860 & 2.638 & 3.1390 & 3.641 & 3.7940 \\
$\mu_{2}$ & -0.4979 & 0.1209 & 0.04 & -0.7369 & -0.6819 & -0.4977 & -0.3163 & -0.2605 \\
$\Sigma_{11}$ & 0.4434 & 1.1000 & 0.37 & 0.0787 & 0.09277 & 0.2627 & 1.245 & 1.8240 \\
$\Sigma_{12}$ & -0.0881 & 0.3623 & 0.12 & -0.4487 & -0.301 & -0.0468 & 0.0212 & 0.0507 \\
$\Sigma_{21}$ & -0.0881 & 0.3623 & 0.12 & -0.4487 & -0.301 & -0.0468 & 0.0212 & 0.0507 \\
$\Sigma_{22}$ & 0.0581 & 0.1555 & 0.06 & 0.0095 & 0.01134 & 0.0341 & 0.1653 & 0.2420 \\
$\sigma$ & 0.0251 & 0.0030 & 0.01 & 0.0201 & 0.02073 & 0.0248 & 0.03045 & 0.0318 \\
\hline
\end{tabular}

interval for the parameter of interest (in OpenBUGS, $\alpha=$ 0.2 ). From the final $T$ iterations we calculate the empirical credible interval for each chain. We then calculate the average width of the intervals across the $L$ chains and denote this by $L$. Finally, we calculate the width $l$ of the empirical credible interval based on all $L T$ samples pooled together. The ratio $\widehat{R}=L / l$ of pooled to average interval widths should be greater than 1 if the starting values are suitably overdispersed; it will also tend to 1 as convergence is approached, and so we might assume convergence for practical purposes if $\widehat{R}<1.05$.

Rather than calculating a single value of $\widehat{R}$, we can examine the behavior of $\widehat{R}$ over iteration-time by performing the above procedure repeatedly for an increasingly large fraction of the total iteration range, ending with all of the final $T$ iterations contributing to the calculation as described above.

In the OpenBUGS software, the bgr diagram can achieve above approach. OpenBUGS automatically chooses the number of iterations between the ends of successive ranges: $\max (100,2 T / 100)$. It then plots $\widehat{R}$ in red, $L$ (pooled) in green, and $l$ (average) in blue. As shown in Figure 6, the red line represents $\widehat{R}$, the green line represents $L$, and $l$ corresponds to blue line.

In this study, three chains for each parameter were generated by OpenBUGS software. The convergence diagnosis schematic diagrams for seven model parameters were shown in Figure 6.

As shown in Figure 6, the convergence feature of model parameters is good, and the trend of model parameter with iteration increase is convergent. In other words, the posterior distributions of seven parameters are accurate and credible.

4.3. Prediction Model of Fatigue Crack Growth. The prediction of crack growth life (period) is a main thesis in the aircraft structural engineering. In this study, it is aimed to accurately predict the fatigue crack growth behavior (curve) for one target specimen using least information.

The Bayesian theorem can be written in model updating context as

$$
P(\vec{\theta} \mid D, M) \propto P(D \mid \vec{\theta}, M) P(\vec{\theta} \mid M)
$$

where $P(\vec{\theta} \mid D, M)$ corresponds to the PDF for the crack growth model $M$ after updating with the crack growth test observations $D$; it is called the PDF of posterior distribution. $P(\vec{\theta} \mid M)$ is the PDF of model parameters $\vec{\theta}$ for the crack growth model $M$ before updating; it is called the PDF of prior distribution. And $P(D \mid \vec{\theta}, M)$ is the likelihood of occurrence of the crack growth observation $D$ given the vector of model parameter $\vec{\theta}$ and crack model $M$.

The fatigue crack growth experimental results can be regarded as the prior information. And the parameter prior distribution of crack growth model (4) can be obtained by above approach. According to the analysis and inference in the Section 4.1, crack growth model parameters $\vec{\theta}=\left(\theta_{1}, \theta_{2}\right)$ are subjected to multinormal $(\mu, \vec{\Sigma})$ :

$$
\begin{aligned}
& \vec{\mu}=(3.1390,-0.4979) \\
& \vec{\Sigma}=\left(\begin{array}{cc}
0.4434 & -0.0881 \\
-0.0881 & 0.0581
\end{array}\right) .
\end{aligned}
$$

Therefore, the posterior likelihood function of model parameters for one target specimen can be calculated as

$$
\begin{aligned}
& P(\vec{\theta} \mid D, M) \\
& \propto \prod_{z=1}^{m} \exp \left(-\frac{1}{2}\left(\frac{y_{z}-\left(1 / \theta_{2}\right) \ln \left(1-a_{0} \theta_{2} \theta_{1} \theta_{2} N_{z}\right)}{\sigma}\right)^{2}\right) \\
& \quad \cdot H(\vec{\theta}),
\end{aligned}
$$

where $m$ is the number of observation point used to update the posterior distribution of model parameters. And if the first test data point was used to update the posterior distribution of parameters $\theta_{1}$ and $\theta_{2}, m=1$.

The posterior distribution can be obtained via using Markov chain Monte Carlo (MCMC) simulation with the combination of the likelihood function (14) with prior distribution (13).

If the model parameters $\theta_{1}$ and $\theta_{2}$ for a specimen are determined, the fatigue crack propagation curve of this specimen can be obtained by (4). As a result, the residual growth life (period) of this specimen can be determined. 


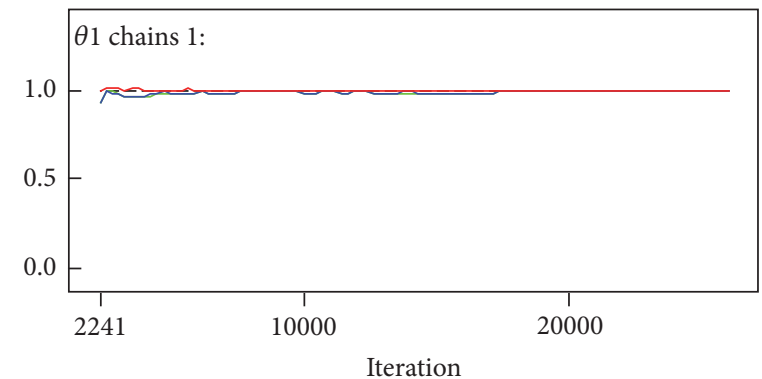

(a)

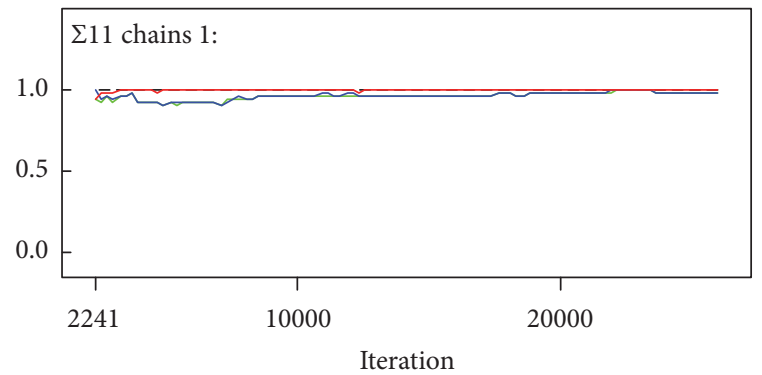

(c)

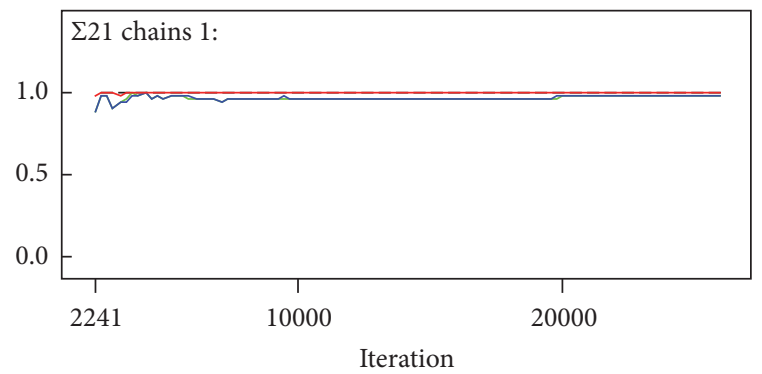

(e)

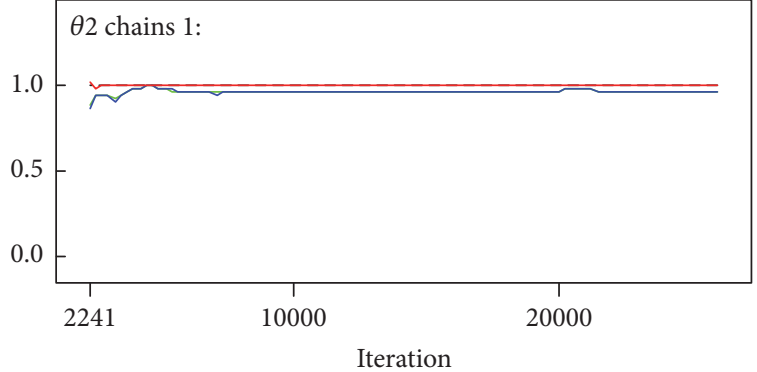

(b)

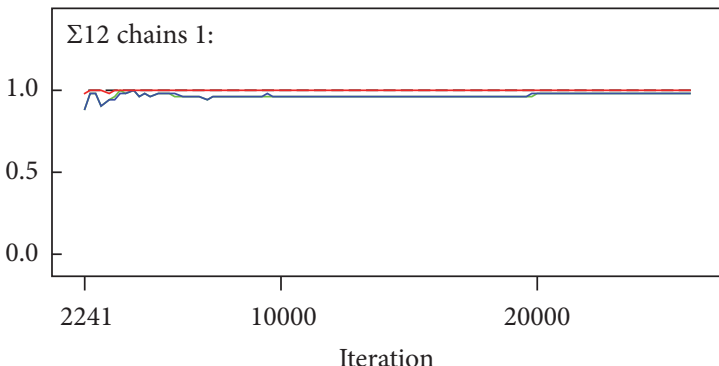

(d)

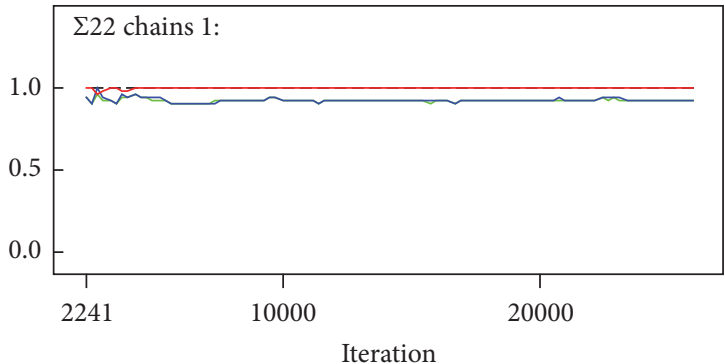

(f)

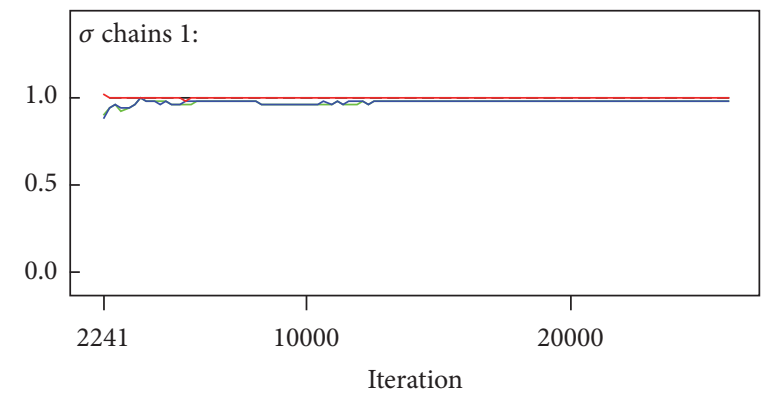

(g)

FiguRE 6: The bgr diagram of convergence diagnosis for fatigue crack growth model parameters: (a) $\theta_{1}$, (b) $\theta_{2}$, (c) $\Sigma_{11}$, (d) $\Sigma_{12}$, (e) $\Sigma_{21}$, (f) $\Sigma_{22}$, and $(\mathrm{g}) \sigma$.

\section{Experimental Validations}

This section presents the validation of the proposed methodology via using crack propagation data from additional crack growth test.

5.1. Additional Crack Growth Test. Additional crack growth test employs the same experimental method, environmental condition, and measurement technology as the test in Section 2. Similarly, when the crack approximately propagates to $0.5 \mathrm{~mm}$, start counting the number of loading cycles. In other words, the crack length is $0.5 \mathrm{~mm}$ when the number of loading cycles is zero.

The specific initial crack length and the crack length after 9000 cycles (number 5, number 6, and number 7 target specimen) are listed in Table 8 . It is noted that the 
TABLE 8: Crack lengths of initialization and first test point (mm).

\begin{tabular}{lcc}
\hline Specimen & Initial crack length & Crack length after 9000 cycles \\
\hline Number 5 & 0.5 & 0.66 \\
Number 6 & 0.53 & 0.74 \\
Number 7 & 0.5 & 0.69 \\
\hline
\end{tabular}

experimental data (in Table 8) can represent the in-service observation during the service for aircraft structures.

5.2. Prediction of Crack Growth. The prediction of crack growth curve (number 5 specimen) combines different test data information (different data sample length and different test data). Mark different information source as Case I, Case II, and Case III. A particular introduction of Case I-Case III is presented as follows.

For Case I, the prior distribution of matrix parameter $\vec{\theta}=\left(\theta_{1}, \theta_{2}\right)$ is the only information source for the prediction of crack growth curve. So the posterior distribution mean of parameter $\vec{\theta}=\left(\theta_{1}, \theta_{2}\right)$ is $(3.1390,-0.4979)$.

For Case II, the information source includes prior distribution of matrix parameter $\vec{\theta}=\left(\theta_{1}, \theta_{2}\right)$ and the first observation data point $(N=9000, a(N)=0.66 \mathrm{~mm})$. And the prediction model of Case II is the proposed model, which corresponds to the Section 4.3. Based on (14), the likelihood of Case II is expressed as

$$
\begin{aligned}
& P(\vec{\theta} \mid D, M) \\
& \quad \propto \exp \left(-\frac{1}{2}\left(\frac{y_{5,1}-\left(\ln \left(1-a_{5,0} \theta_{5,2} \theta_{5,1} \theta_{5,2} N_{1}\right) / \theta_{5,2}\right)}{\sigma}\right)^{2}\right) \\
& \cdot H(\vec{\theta}) .
\end{aligned}
$$

Based on the above likelihood function of posterior distribution and the OpenBUGS package, the mean value of posterior distribution of matrix parameters $\vec{\theta}=\left(\theta_{1}, \theta_{2}\right)$ can be obtained. Through the MC-error control and the convergence diagnose of the posterior distribution of the matrix parameter $\vec{\theta}$, the mean value is $(2.6035,-0.3172)$.

For Case III, the first observation data $(N=9000, a(N)=$ $0.66 \mathrm{~mm}$ ) is the only information source for the prediction of crack growth curve. The posterior distribution mean value of $\vec{\theta}=\left(\theta_{1}, \theta_{2}\right)$ is $(0.8838,-2.4941)$, which can be estimated by using the method of Section 3.2.

For number 6 and number 7 specimen, they are also deviated to Case I, Case II, and Case III. The posterior distribution mean values of matrix parameters $\vec{\theta}=\left(\theta_{1}, \theta_{2}\right)$ for the additional three target specimens are listed in Table 10.

5.3. Additional Experimental Observation. The additional fatigue crack growth tests to 90000 loading cycles were conducted. The crack growth lengths of number 5 , number 6 , and number 7 specimens are listed in Table 9 (include the data of Table 8).

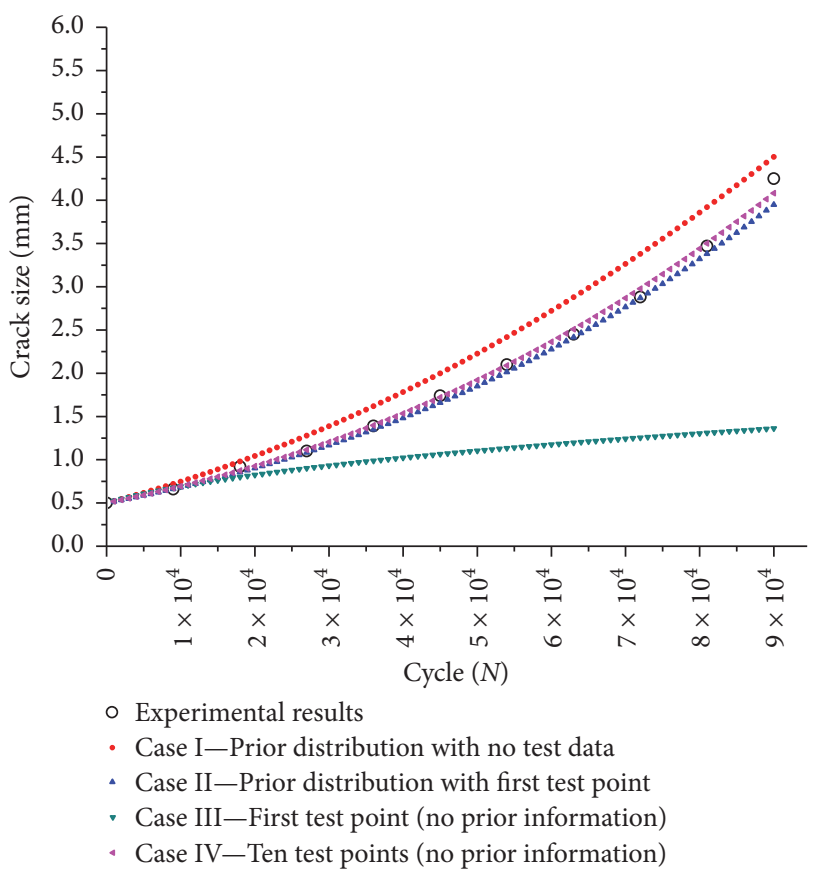

FIGURE 7: Crack propagation prediction curves of number 5 specimen.

The parameters are fitted with the same method of Section 3.2. And the information source is called Case IV. The posterior distribution of fitting parameters $\left(\theta_{1}, \theta_{2}\right)$ is listed in Table 10.

The model parameter posterior distribution mean values of Case I-Case IV for number 5, number 6, and number 7 specimens are listed in Table 10.

The fatigue crack growth curve can be obtained by substituting the parameter of posterior distribution mean value to crack growth model (4). In this paper, for one target specimen, we can obtain four fatigue crack growth curves, corresponding to the above Case I, Case II, Case III, and Case IV, respectively. For example, four fatigue crack growth curves and experimental $a-N$ observations of number 5 specimen are shown in Figure 7.

As shown in Figure 7, the fatigue crack growth curve of Case III is far away from the experimental $a-N$ observations. And the absolute error between fatigue crack growth curve of Case II and experimental $a-N$ observations is smaller than that of Case III. Compare Case II with Case III, we can obtain the prior information that is very important for the prediction of fatigue crack growth behavior.

The fatigue crack growth curves for number 6 and number 7 specimens are obtained by the same method and analysis steps, which are shown in Figures 8 and 9. And the experimental results of number 6 and number 7 specimen are shown in Figures 8 and 9, respectively.

As shown in Figures 7, 8, and 9, we can know that the prediction of fatigue crack growth of Case III is far away from the experimental $a-N$ observations, so the error cannot satisfy the requirements of aircraft engineering. 
TABLE 9: Crack lengths for different cycles (mm).

\begin{tabular}{|c|c|c|c|c|c|c|c|c|c|c|c|}
\hline \multirow{2}{*}{ Specimen } & \multicolumn{11}{|c|}{ Cycle } \\
\hline & 0 & 9000 & 18000 & 27000 & 36000 & 45000 & 54000 & 63000 & 72000 & 81000 & 90000 \\
\hline Number 5 & 0.5 & 0.66 & 0.92 & 1.1 & 1.39 & 1.74 & 2.1 & 2.45 & 2.88 & 3.47 & 4.25 \\
\hline Number 6 & 0.55 & 0.77 & 1.02 & 1.2 & 1.51 & 2 & 2.49 & 2.96 & 3.59 & 4.3 & 5.41 \\
\hline Number 7 & 0.5 & 0.70 & 0.93 & 1.16 & 1.53 & 1.96 & 2.42 & 2.74 & 3.3 & 4.12 & 4.84 \\
\hline
\end{tabular}

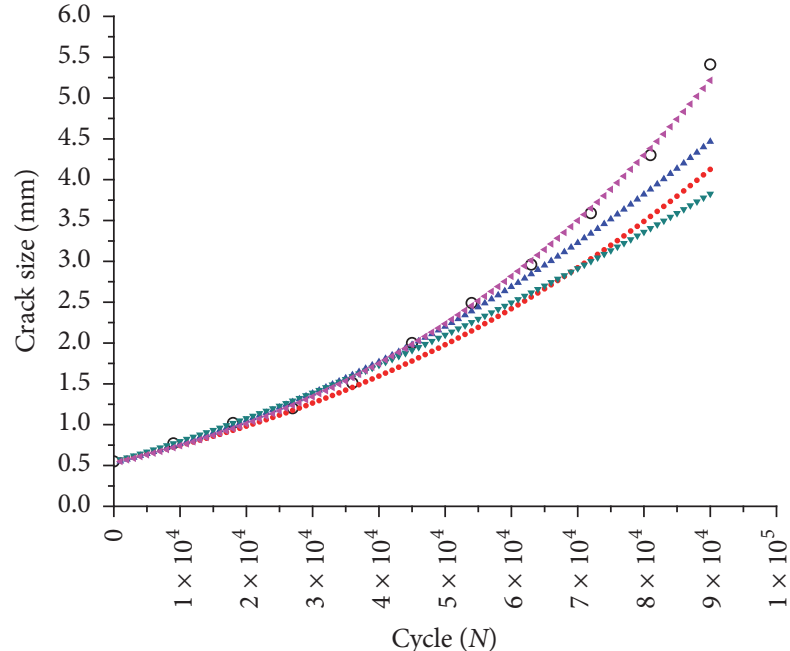

- Experimental results

- Case I-Prior distribution with no test data

- Case II-Prior distribution with first test point

- Case III-First test point (no prior information)

4 Case IV-Ten test points (no prior information)

FIGURE 8: Crack propagation curves prediction of number 6 specimen.

Comparative analysis of the prediction for the fatigue crack growth of Figures 7, 8, and 9 is based on the crack growth model parameters prior distribution and observation data (Case II) and we can obtain the crack growth curve accurately. Therefore, the prediction method (Case II) proposed in this study is an advanced technology.

5.4. Error Analysis. Due to the high error of Case III, it will not be considered in the following analysis. The absolute errors (prediction value to experimental observations value) of Case I, Case II, and Case IV are analyzed in the following steps. And the absolute error is defined as

absolute error

$$
\begin{aligned}
= & \left|\frac{(\text { pridiction value })-(\text { experiment value })}{\text { experiment value }}\right| \\
& \times 100 \% .
\end{aligned}
$$

Figure 10 shows the prediction crack life (Figure 7) versus the experimental results (Table 9) of number 5 target specimen.

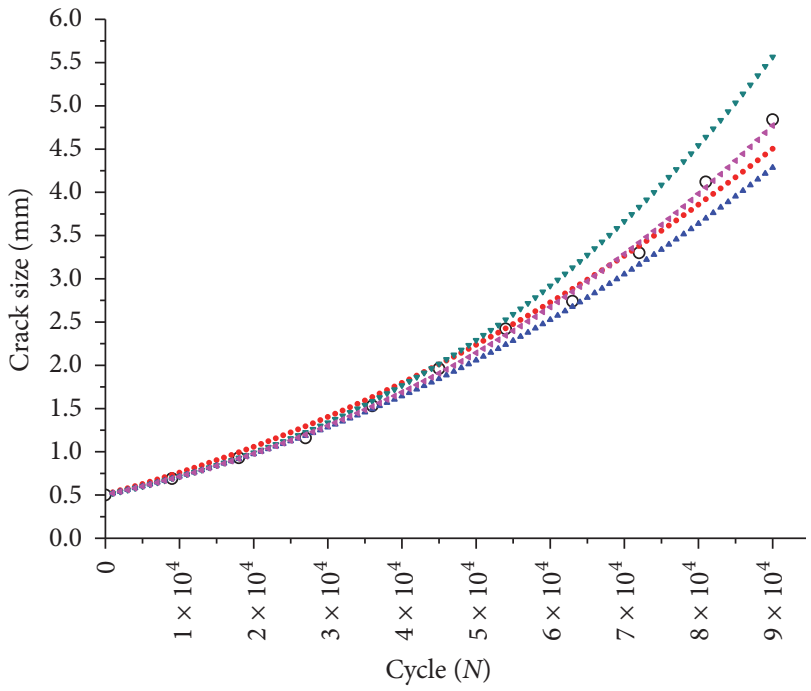

- Experimental results

- Case I-Prior distribution with no test data

- Case II-Prior distribution with first test point

- Case III-First test point (no prior information)

- Case IV-Ten test points (no prior information)

FIGURE 9: Crack propagation curves prediction of number 7 specimen.

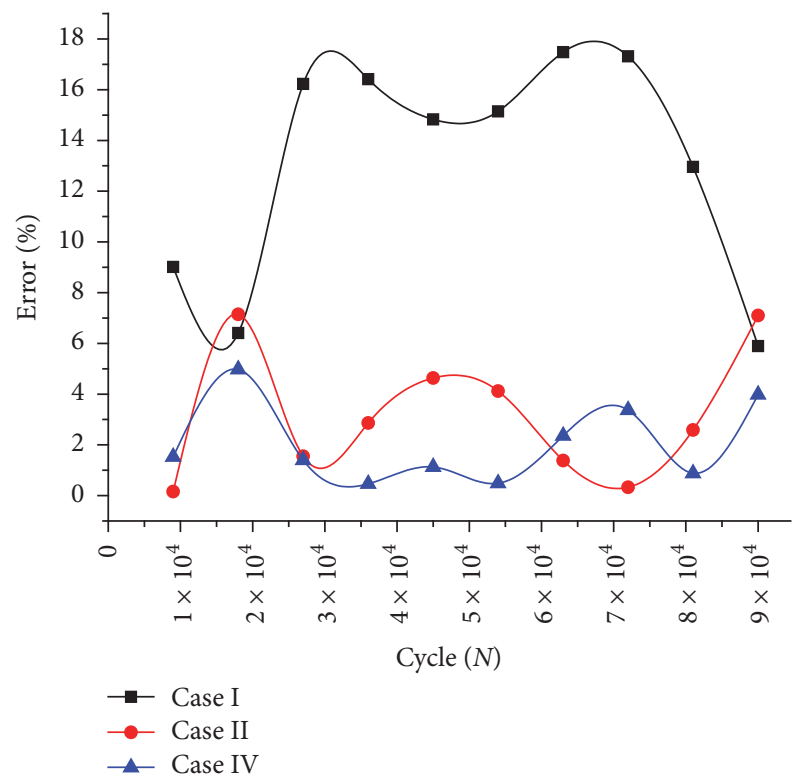

FIGURE 10: Prediction error of crack propagation curve for number 5 specimen. 


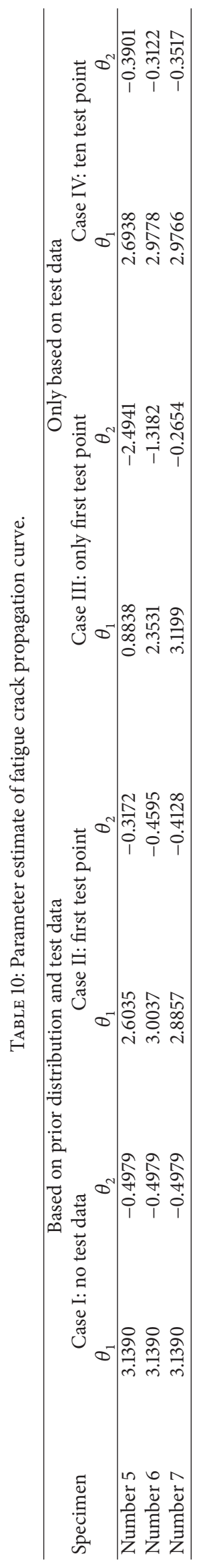




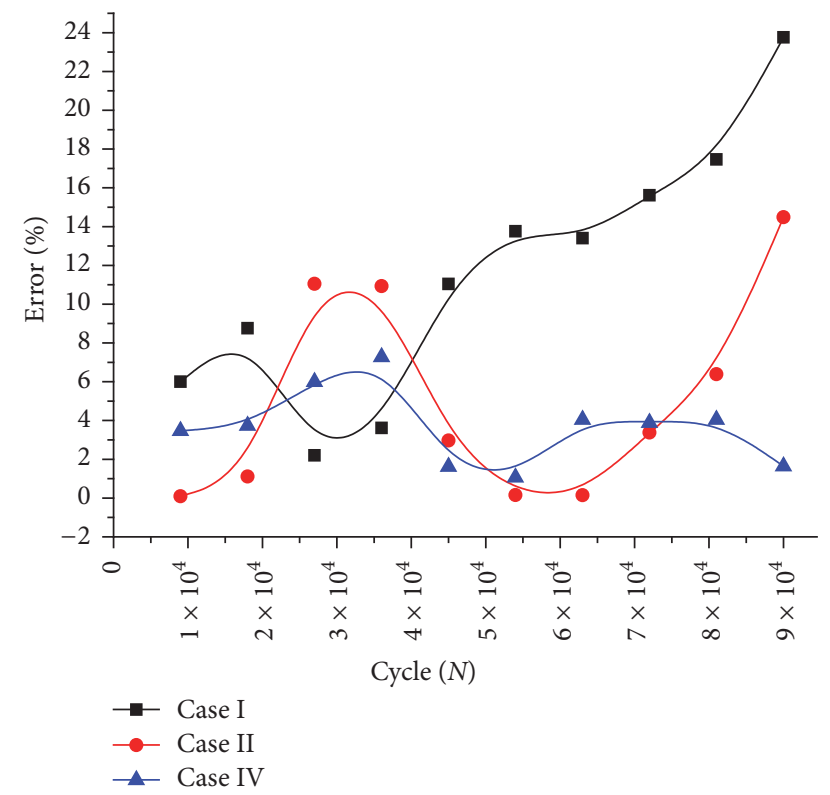

FIGURE 11: Prediction error of crack propagation curve for number 6 specimen.

As shown in Figure 10, the absolute error of Case IV is the smallest for the three cases. In other words, the prediction curve (life) of Case III is in good agreement with experimental observations. Based on the absolute error condition of Case I, it can be known that using the average $a-N$ curve of number 1 , number 2 , number 3 , and number 4 specimens to represent crack growth curve of number 5 specimen may cause bigger error.

Crack life prediction (Figures 8 and 9) versus the experimental results (Table 8) for number 6 and number 7 specimens is shown in Figures 11 and 12, respectively.

As shown in Figures 10, 11, and 12, the absolute error of Case II can satisfy the aircraft engineering accurate needs.

It is worth noting that Case I represents the mean of experimental results. In some time, the prediction of Case I may be more concise than Case II, but it will not happen all the time. In other words, it is a special circumstance, and it is determined by the stochastic and the scatter of fatigue crack growth behavior. For instance, the prediction fatigue crack growth curve of Case I (number 7 specimen, in Figure 12) is in good agreement with the experimental results.

\section{Conclusion and Discussion}

The paper presents a method of fatigue crack propagation by using the crack test information and in-service inspection information. The fatigue crack growth curve of 2024-T62 aluminum alloy is predicted by Bayesian theory with MCMC algorithm and OpenBUGS package. Based on the current investigation, four conclusions are drawn.

(1) The introduced model (4) can describe the fatigue crack growth behavior of 2024-T62 aluminum alloy accurately.

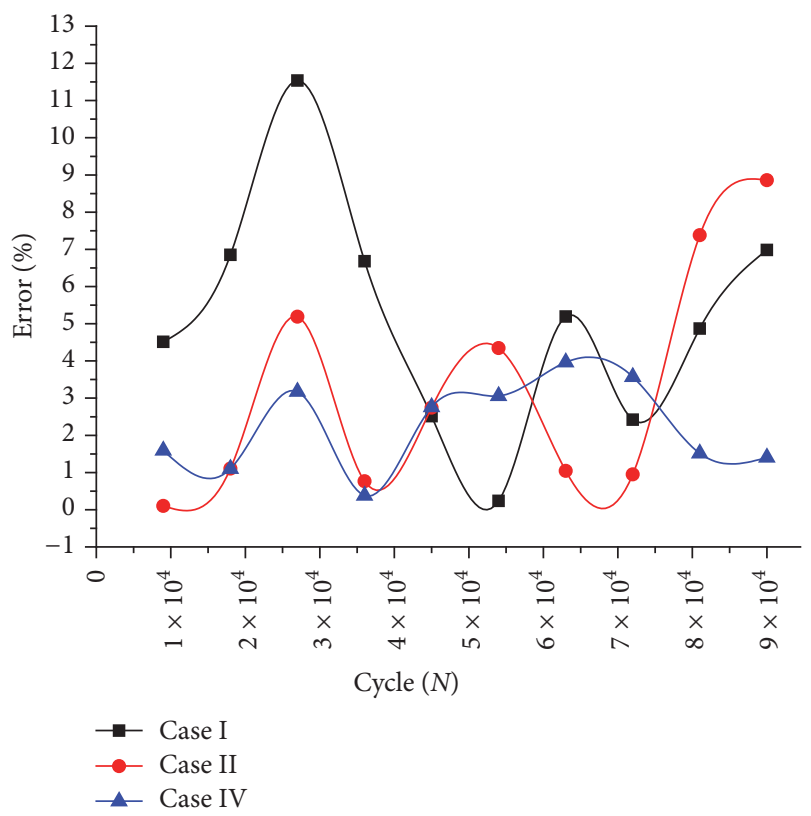

FIGURE 12: Prediction error of crack propagation curve for number 7 specimen.

(2) The scatter of crack length (under same loading cycles) gradually increases with crack propagation process.

(3) Prior information of crack growth model parameter is an important information source to predict the crack growth behavior. And the MCMC is a good method to resolve the statistics estimation issue of multiparameter.

(4) The proposed approach provides an insight into fatigue crack growth curve prediction and associates the MCMC with OpenBUGS package. The predicted fatigue crack growth curve and residual life are in good agreement with the experimental observations. And the performance of proposed approach shows the superior results to the mean value of $a-N$ data.

\section{Conflicts of Interest}

The authors declare no conflicts of interest regarding the publication of this paper.

\section{Acknowledgments}

This work is supported by the National Natural Science Foundation of China (Project no. 51505495). The authors gratefully appreciate the support.

\section{References}

[1] W. F. Wu and C. C. Ni, "Probabilistic models of fatigue crack propagation and their experimental verification," Probabilistic Engineering Mechanics, vol. 19, no. 3, pp. 247-257, 2004.

[2] X. L. Zou, "Statistical moments of fatigue crack growth under random loading," Theoretical and Applied Fracture Mechanics, vol. 39, no. 1, pp. 1-5, 2003. 
[3] W. Li, T. Sakai, Q. Li, and P. Wang, "Statistical analysis of fatigue crack growth behavior for grade B cast steel," Materials and Design, vol. 32, no. 3, pp. 1262-1272, 2011.

[4] H. Y. Liou, C. C. Ni, and W. F. Wu, "Scatter and statistical analysis of fatigue crack growth data," Journal of Chinese Society of Mechanical Engineers, vol. 22, no. 5, pp. 399-407, 2001.

[5] J.-K. Kim and D.-S. Shim, "Variation in fatigue crack growth due to the thickness effect," International Journal of Fatigue, vol. 22, no. 7, pp. 611-618, 2000.

[6] W. F. Wu and C. C. Ni, "A study of stochastic fatigue crack growth modeling through experimental data," Probabilistic Engineering Mechanics, vol. 18, no. 2, pp. 107-118, 2003.

[7] R. Rastogi, S. Ghosh, A. K. Ghosh, K. K. Vaze, and P. K. Singh, "Fatigue crack growth prediction in nuclear piping using Markov chain Monte Carlo simulation," Fatigue and Fracture of Engineering Materials and Structures, vol. 40, no. 1, pp. 145-156, 2017.

[8] W. R. Gilks, S. Richardson, and D. J. Spiegelhalter, Markov Chain Monte Carlo in Practice, Interdisciplinary Statistics, Chapman \& Hall/CRC, London, UK, 1996.

[9] Standard A. S. T. M., "E647, Standard test method for measurement of fatigue crack growth rates," Annual Book of ASTM Standards, Section Three: Metals Test Methods and Analytical Procedures, vol. 3, pp. 628-670, 2002.

[10] N. E. Dowling, "Mechanical behavior of materials: engineering methods for deformation," Fracture, and Fatigue, Pearson, 2012.

[11] N. Pugno, M. Ciavarella, P. Cornetti, and A. Carpinteri, "A generalized Paris' law for fatigue crack growth," Journal of the Mechanics and Physics of Solids, vol. 54, no. 7, pp. 1333-1349, 2006.

[12] M. S. Hamada, A. Wilson, C. S. Reese, and H. Martz, Bayesian Reliability, Springer Science \& Business Media, New York, 2008.

[13] K. Mosegaard and A. Tarantola, "16 Probabilistic approach to inverse problems," International Geophysics, vol. 81, pp. 237-265, 2002.

[14] M. Amiri and M. Modarres, "Short fatigue crack initiation and growth modeling in aluminum 7075-T6," Journal of Mechanical Engineering Science, vol. 229, no. 7, pp. 1206-1214, 2015.

[15] D. Spiegelhalter, A. Thomas, N. Best et al., OpenBUGS user manual, version 3.0.2, MRC Biostatistics Unit, Cambridge, 2007.

[16] A. G. Gelman and D. B. Rubin, "Inference from iterative simulation using multiple sequences," Statistical Science, vol. 7, no. 4, pp. 457-472, 1992.

[17] S. P. Brooks and A. Gelman, "General methods for monitoring convergence of iterative simulations," Journal of Computational and Graphical Statistics, vol. 7, no. 4, pp. 434-455, 1998. 


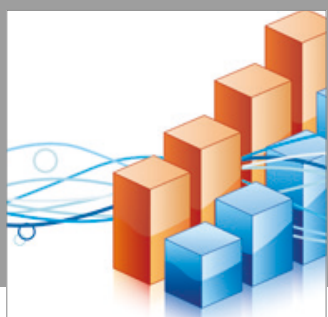

Advances in

Operations Research

vatersals

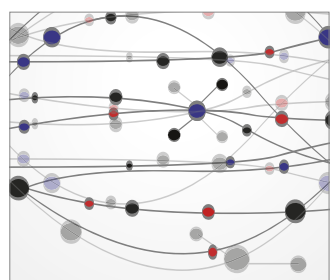

\section{The Scientific} World Journal
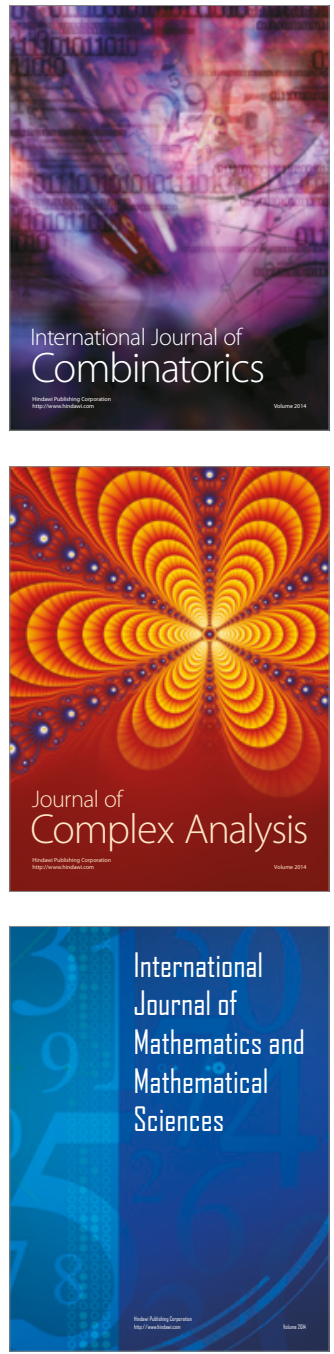
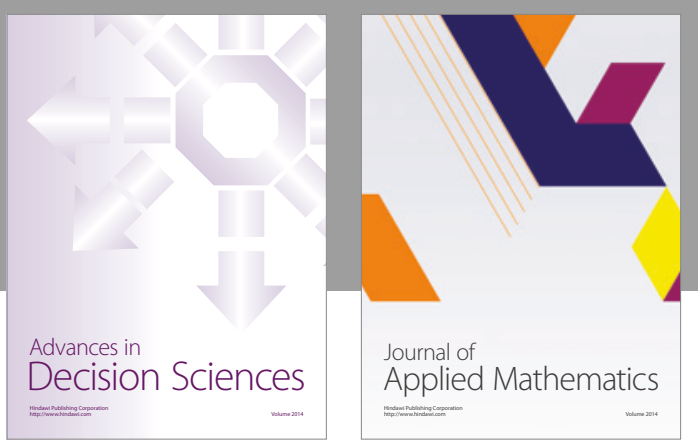

Algebra

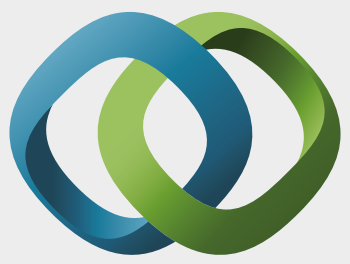

\section{Hindawi}

Submit your manuscripts at

https://www.hindawi.com
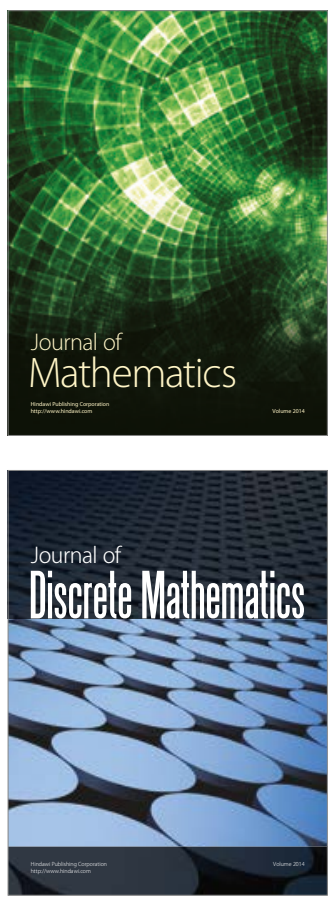

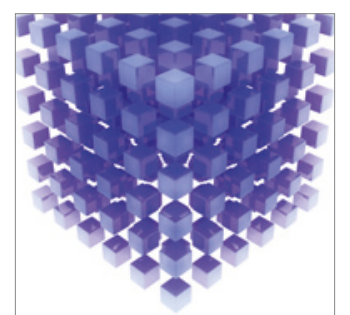

Mathematical Problems in Engineering
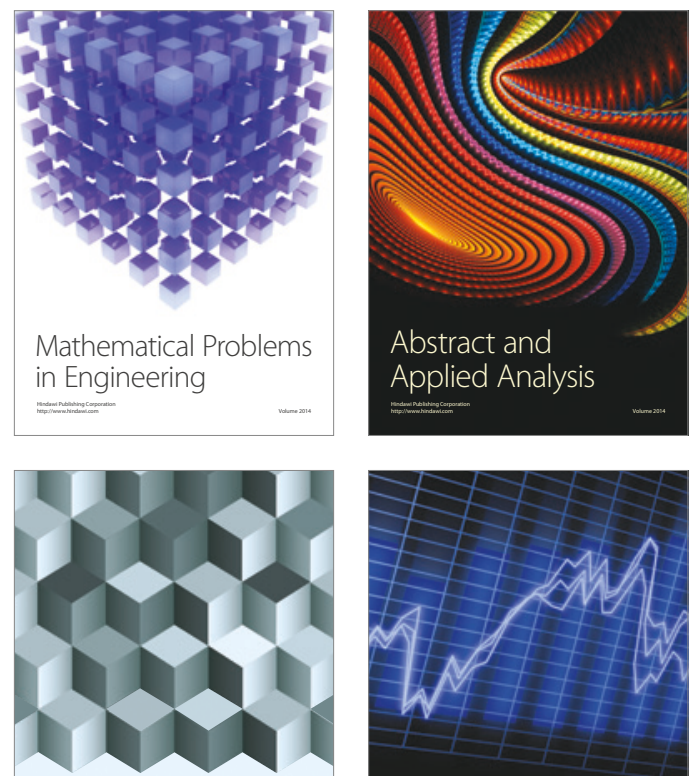

Journal of

Function Spaces

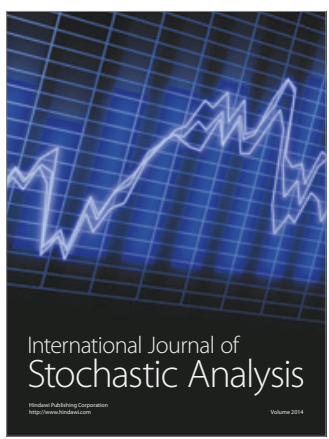

Probability and Statistics
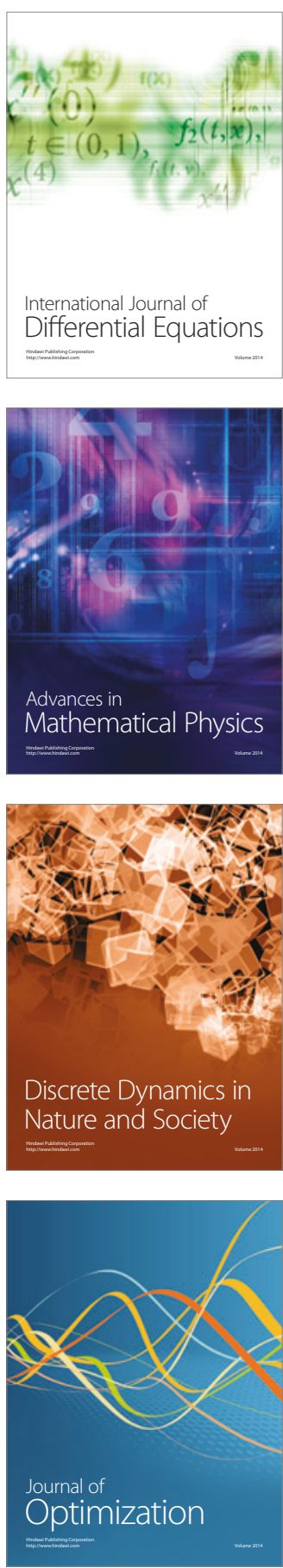